\title{
1 Predicting and measuring decision rules for social recognition in a Neotropical frog
}

3 James P. Tumulty ${ }^{\mathrm{a},}{ }^{*},{ }^{\dagger}$, Chloe A. Fouilloux ${ }^{\mathrm{a}, \mathrm{b}}$, Johana Goyes Vallejos ${ }^{\mathrm{c}}$, Mark A. Bee ${ }^{\mathrm{a}, \mathrm{d}}$

$5 \quad{ }^{a}$ Department of Ecology, Evolution, and Behavior, University of Minnesota, St. Paul, MN, USA

$6 \quad{ }^{b}$ Department of Biology and Environmental Science, University of Jyväskylä, Jyväskylä, Finland

$7 \quad{ }^{\mathrm{c}}$ Division of Biological Sciences, University of Missouri, Columbia, MO, USA

$8 \quad{ }^{\mathrm{d}}$ Graduate Program in Neuroscience, University of Minnesota, Minneapolis, MN, USA

9

$10 \quad{ }^{*}$ Corresponding author. E-mail address: james.tumulty@gmail.com

$11{ }^{\dagger}$ Current address: Department of Neurobiology and Behavior, Cornell University, Ithaca, NY, USA.

12

13 Keywords: social recognition, acoustic communication, signal detection theory, acceptance threshold

14 model, dear enemy effect, decision rules, rocket frog. 


\section{Abstract}

16 Many animals use signals, such as vocalizations, to recognize familiar individuals. However, animals risk

17 making recognition mistakes because the signal properties of different individuals often overlap due to

18 within-individual variation in signal production. To understand the relationship between signal variation

19 and decision rules for social recognition, we studied male golden rocket frogs, which recognize the calls

20 of territory neighbors and respond less aggressively to a neighbor's calls than to the calls of strangers. We

21 quantified patterns of individual variation in acoustic properties of calls and predicted optimal

22 discrimination thresholds using a signal detection theory model of receiver utility that incorporated signal

23 variation, the payoffs of correct and incorrect decisions, and the rates of encounters with neighbors and

24 strangers. We then experimentally determined thresholds for discriminating between neighbors and

25 strangers using a habituation-discrimination experiment with territorial males in the field. Males required

26 a threshold difference between $9 \%$ and $12 \%$ to discriminate between calls differing in temporal

27 properties; this threshold matched those predicted by a signal detection theory model under ecologically

28 realistic assumptions of infrequent encounters with strangers and relatively costly missed detections of

29 strangers. We demonstrate empirically that receivers group continuous variation in vocalizations into

30 discrete social categories and show that signal detection theory can be applied to investigate evolved

31 decision rules. 


\section{Introduction}

33 Animals must frequently make decisions about how to categorize natural variation in behaviorally

34 relevant stimuli (Guilford and Dawkins 1991; Shettleworth 2010; Miller and Bee 2012). Such

35 categorization underlies the use of communication signals for social recognition (Colgan 1983; Wiley

$362013 a$ ), the phenomenon by which animals recognize individuals they have previously interacted with

37 and assign them to social categories (e.g., "neighbor", "offspring", or "nest-mate"). For example, the use

38 of vocalizations to recognize familiar individuals has been demonstrated in a variety of vertebrate taxa,

39 including birds (Brooks and Falls 1975a; Aubin and Jouventin 2002), mammals (Cheney and Seyfarth

40 1980; Proops et al. 2009), amphibians (Davis 1987; Chuang et al. 2017), and fish (Myberg and Riggio

41 1985). Social recognition allows animals to direct behaviors to appropriate individuals but is subject to

42 uncertainty because signal properties, such as the acoustic properties of vocalizations, often vary both

43 among and within individuals (Gerhardt 1991). For recognition to be possible based on such signals,

44 signal properties must be individually distinctive, meaning they vary less within individuals than among

45 individuals (Beecher 1989). Receivers must then group within-individual variation in signal properties

46 into discrete social categories and possess decision rules for behaviorally discriminating between signals

47 produced by individuals belonging to these different categories. Despite many examples of social

48 recognition (Tibbetts and Dale 2007; Wiley 2013a; Carlson et al. 2020), we still know little about how

49 animals categorize variation in signal properties to accomplish this task (Miller and Bee 2012; Yorzinski

50 2017; Tumulty and Sheehan 2020).

51 Because communication-based social recognition is a product of coevolution between signalers

52 and receivers, a receiver's decision rules should reflect the natural variation in signals that occurs within

53 and among individuals (Nelson 1989; Nelson and Marler 1990; Bee and Gerhardt 2001a, 2001b, 2001c;

54 Aubin and Jouventin 2002; Bee 2004). However, the signal properties of different individuals often

55 overlap, and animals aiming to discriminate between individuals based on these signal properties risk

56 making mistakes. Additionally, the outcomes of correct and incorrect decisions may have different

57 payoffs (in terms of fitness), and animals may differentially incur these payoffs based on the rates at 
58 which they encounter different individuals. These three factors - (1) individual variation in signals, (2)

59 payoffs of correct and incorrect decisions, and (3) encounter rates - have likely shaped decision rules

60 through evolution by natural selection and are thus fundamental considerations in applying signal

61 detection theory to investigate the evolution of social recognition (Reeve 1989; Wiley 1994, 2006,

62 2013b). Signal detection theory and the associated acceptance threshold model (Reeve 1989) have been

63 influential in interpreting adaptive decision rules in animals (e.g., Getty 1995; Lynn et al. 2005; Hauber et

64 al. 2006; Steiger and Müller 2010; Mora-Kepfer 2014; Hanley et al. 2019; Rossi et al. 2019). However,

65 studies typically rely on assumptions about the underlying distributions of cue or signal variation rather

66 than measuring them directly to make precise predictions about decision rules.

To investigate the relationship between signal variation and decision rules for social recognition, we analyzed call variation and measured decision rules for discriminating between territory neighbors and strangers in golden rocket frogs, Anomaloglossus beebei. Male golden rocket frogs defend territories in respond less aggressively to the calls of neighbors than to the calls of strangers (Bourne et al. 2001;

73 Tumulty and Bee 2021), producing a phenomenon called the "dear enemy effect" (Wilson 1975; Tumulty

74 2018). This behavior should allow males to reduce the costs of contests (Dyson et al. 2013) that would otherwise be associated with repeated aggressive interactions with established neighbors (Getty 1987; Temeles 1994). The evolution of neighbor recognition and the dear enemy effect in golden rocket frogs

77 has not been associated with an increase in the identity information in signals; the advertisement calls of

78 golden rocket frogs have similar patterns of among- and within-individual variation as those of a close

79 relative (Anomaloglossus kaiei) that does not discriminate between the calls of neighbors and strangers

80 (Tumulty et al. 2021). Instead, selection has acted on receivers in golden rocket frogs, likely by modifying

81 pre-existing mechanisms of plasticity in aggression to create decision rules that are specific to the calls of

82 individual neighbors (Tumulty et al. 2021). The decision rules that males use to categorize calls as

83 belonging to neighbors or strangers are currently unknown. In essence, a territorial male must decide 
84 whether an aggressive response to the call of a nearby male is warranted based on whether the call is

85 sufficiently different from their memory of the acoustic properties of a neighbor's calls. Such thresholds

86 for discrimination place boundaries around perceptual categories and are sometimes referred to as "just-

87 meaningful differences" (Nelson 1988; Nelson and Marler 1990; Gerhardt 1992). In contrast to “just-

88 noticeable differences," which describe the smallest perceptible differences, just-meaningful differences

89 refer to the smallest ecologically meaningful difference in a signal property, and they can be empirically

90 measured in playback studies as the smallest difference in a property that elicits a differential response

91 from a receiver. Although the signal properties mediating recognition are unknown, temporal properties

92 related to the call's pulsatile structure are the most reliable for statistically discriminating between

93 individuals based on calls (Pettitt et al. 2013; Tumulty et al. 2021).

94 This study had three objectives. First, we generated predictions about the optimal thresholds for

95 discriminating between neighbors and strangers based on patterns of individual variation in the acoustic

96 properties of advertisement calls, focusing on the most individually distinctive properties of pulse

97 temporal structure. To do so, we analyzed acoustic variation in advertisement calls and used a signal

98 detection theory model of receiver utility to predict optimal thresholds based on call variation. Second, we

99 measured the discrimination thresholds of territorial males using a field playback experiment based on the

100 habituation-discrimination paradigm (Rankin et al. 2009; Shettleworth 2010). Habituation is a widespread

101 form of learning that underlies plasticity of aggression in frogs (Brenowitz and Rose 1994; Owen and

102 Perrill 1998; Bee and Gerhardt 2001c, 2001b, 2002; Marshall et al. 2003; Humfeld et al. 2009; Tumulty et

103 al. 2021) and has been suggested as a learning mechanism underlying neighbor-stranger discrimination in

104 territorial species (Peeke and Peeke 1973; Peeke 1984; Petrinovich 1984). Males initially responded

105 aggressively to calls simulating the arrival of a new neighbor by approaching the speaker and producing

106 aggressive calls. We repeatedly played the stimulus until males met pre-determined habituation criteria

107 indicating they were no longer responding aggressively to playbacks. Following habituation, we changed

108 the temporal properties of pulses within stimulus calls by magnitudes spanning the range of values

109 predicted by our signal detection theory model to empirically estimate the threshold change required to 
110 elicit a recovery of habituated aggression. A recovery of aggression would indicate that a territorial male

111 behaviorally discriminated between the habituation and discrimination stimuli based on the

112 experimentally manipulated properties and that it treated the discrimination stimulus as if it were a call

113 produced by a stranger. Finally, we used the signal detection theory model to explore the combinations of

114 stranger encounter rates and payoffs for correct and incorrect decisions that would produce the observed

115 discrimination thresholds from our playback experiment.

\section{Methods}

Study system

120 Golden rocket frogs are a species of poison frog (Aromabatidae, Dendrobatoidea) endemic to Guyana,

121 South America. Playback experiments were performed from June-July 2017 in Kaieteur National Park,

122 Guyana, where these frogs live and breed in large terrestrial bromeliads (Brocchinia micrantha) that grow

123 along the plateau near Kaieteur Falls. Males aggressively defend stable territories that consist of one or

124 more bromeliads (Tumulty and Bee 2021). These plants contain reproductive resources in the form of

125 phytotelmata, which are pools of water that collect in leaf axils and serve as oviposition and tadpole

126 deposition sites (Bourne et al. 2001; Pettitt et al. 2018; Tumulty and Bee 2021). Males care for eggs and

127 tadpoles in their territories, attending egg clutches and transporting tadpoles between phytotelmata

128 (Bourne et al. 2001; Pettitt et al. 2020). Golden rocket frogs are diurnal, and males regularly vocalize

129 from their territories in the morning with conspicuous advertisement calls. These calls consist of 1-6 short

130 pulses (typically 2-4 pulses) with dominant frequencies between 4.6 and $5.8 \mathrm{kHz}$ (Fig. 1b, c) and are

131 typically delivered at rates of about 24 calls/min (Bourne et al. 2001; Pettitt et al. 2012; Narins et al.

132 2018). Territorial males respond aggressively to playbacks of conspecific advertisement calls with

133 aggressive calls and phonotaxis (Bourne et al. 2001; Tumulty and Bee 2021).

135 Acoustic recordings and measurements 
136 We recorded advertisement calls of territorial males from a distance of 0.5-1 m using a microphone

137 (Sennheiser ME-66, Wedemark, Germany) and digital recorder (Marantz PMD-620, Kanagawa, Japan;

$13844.1 \mathrm{kHz}$ sampling rate, 16-bit resolution). After recording, we measured the air temperature at the male's

139 calling site. Some acoustic properties of frog calls can vary with temperature (reviewed in Gerhardt and

140 Huber 2002), but there was very little temperature variation during the times when this species regularly

141 calls (range $=22.8$ to $25.8^{\circ} \mathrm{C}$ for our recordings). We measured the temporal and spectral properties of 20

142 advertisement calls from each of 20 males (400 calls total) using Raven Pro 1.5 (Cornell Lab of

143 Ornithology, Ithaca, NY). Acoustic properties of each pulse in a call were measured separately. We

144 measured call duration as the time from the onset of the first pulse to the offset of the last pulse in a call,

145 and the call interval as the time from the offset of the last pulse in a call to the onset of the first pulse in

146 the subsequent call. Call period was the sum of call duration and call interval. For each pulse in a call, we

147 measured pulse duration as the time between the onset and the offset of the pulse and pulse interval as the

148 time between the offset of the pulse and the onset of the subsequent pulse. The sum of pulse duration and

149 pulse interval was pulse period. Additionally, we measured two temporal properties to describe pulse

150 shape: pulse rise time (onset to maximum amplitude) and pulse fall time (maximum amplitude to offset).

151 We measured the dominant frequency of each pulse using the max frequency function on the power

152 spectrum of the pulse (1024 point, Hamming window). 
(a)
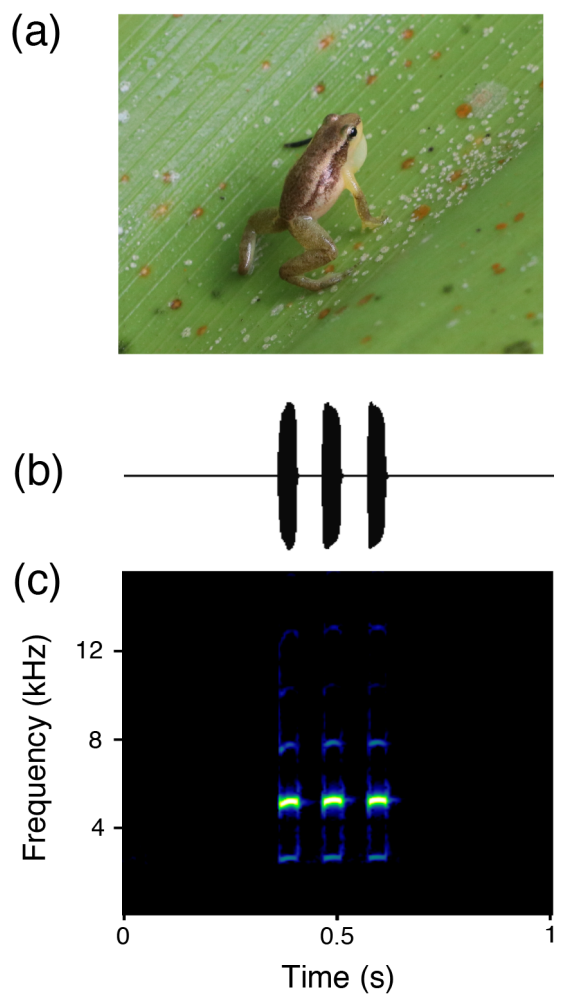

(d)

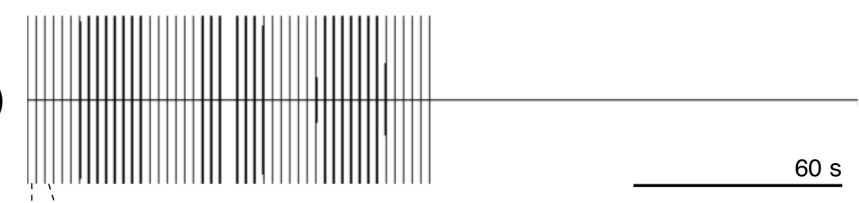

(e)



(f)

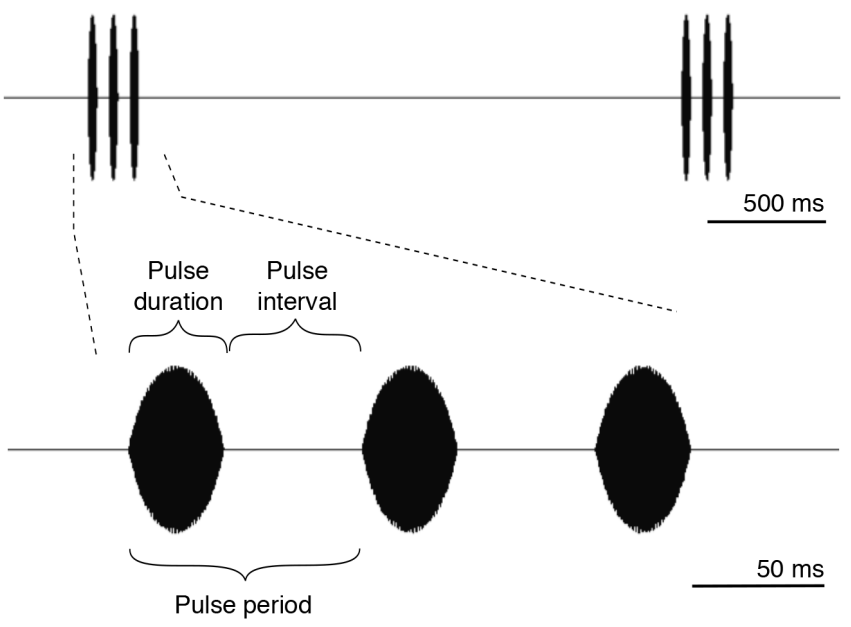

155 Figure 1. Natural and synthetic vocalizations of male golden rocket frogs. (a) Male golden rocket frogs

156 are small (16-18mm) poison frogs that call and defend territories in large terrestrial bromeliads. (b)

157 Waveform (amplitude over time) and (c) spectrogram (frequency over time) of a three-pulse

158 advertisement call produced by a male golden rocket frog. (d-f) Waveforms of a synthetic advertisement

159 call stimulus used in habituation-discrimination playbacks. (d) The full 4-minute stimulus period

160 consisted of 2 minutes of advertisement calls followed by 2 minutes of silence. (e) A close-up of two

161 consecutive advertisement calls showing the temporal properties of calls, and (f) a close-up of one

162 advertisement call showing the three pulse temporal properties that were manipulated in our experiment.

164 Predicting optimal decision rules

165 To predict optimal thresholds for discriminating between neighbors and strangers, we applied the logic of

166 signal detection theory to the dear enemy effect. In doing so, we asked what threshold would be most

167 useful for a territory owner aiming to detect the "signal" of a stranger's calls while ignoring the "noise" of 
168 a neighbor's calls. A territory owner must first form a memory of the acoustic properties of a neighbor's

169 calls as a result of repeatedly hearing the neighbor vocalize. This memory must be broad enough to

170 accommodate natural levels of within-individual variation in calls. Then, upon hearing a new call, the

171 territory owner must decide if the call is sufficiently different from its memory of its neighbor's calls that

172 the call is more likely to have been produced by a stranger than by a neighbor. Thus, the "noise" that

173 males must ignore can be represented as the distribution of within-individual differences that occur in

174 advertisement calls, while the "signal" they must detect can be represented by the distribution of among-

175 individual differences. The territory owner must employ a decision rule for determining whether any

176 difference between a call it just heard and its memory of a neighbor's call is more likely due to a within-

177 individual difference that can be ignored or to an among-individual difference that warrants an aggressive

178 response.

179 We generated distributions of within-individual and among-individual differences in acoustic

180 properties using our data set of measurements of call properties. We restricted our analysis to the first

181 three pulses in each call. This decision allowed us to include all 20 individuals in all analyses, as six

182 males never produced calls with more than three pulses. Indeed, in golden rocket frogs, the majority of

183 advertisement calls consist of either two or three pulses ( $73 \%$ of calls in our data set) and calls with more

184 than four pulses are exceedingly rare (Bourne et al. 2001; Pettitt et al. 2012, 2020). While the number of

185 pulses per call can be variable, even within bouts of calling, the acoustic properties of different pulses

186 within calls are generally highly correlated with each other (Fig. S-1). Moreover, Pettitt et al. (2012)

187 reported that analyses of even a single pulse captured magnitudes of within-individual and among-

188 individual differences equivalent to those based on analyses of three pulses. Thus, we could be confident

189 that the first three pulses in calls would capture the meaningful within- and among-individual variation in

190 calls. Prior to analysis we removed any temperature-dependent variation in acoustic properties by using

191 linear regression (Platz and Forester 1988) to standardize values of all call properties to the mean

192 temperature at which recordings were made $\left(24^{\circ} \mathrm{C}\right)$. Such standardization is common in studies of frog

193 vocal behavior (Gerhardt and Huber 2002) and allowed us to remove any variation that was due to a 
194 known environmental influence on frog calls and not due to the individual (phenotypic) differences of

195 interest. Analyses based on acoustic properties without temperature correction yielded very similar results

196 and can be found in the supplementary material (Tables S-1, S-2, S-3, S-4). For each temperature-

197 standardized property, we computed pairwise differences (as a percentage of the smaller value) between

198 every call and every other call in the data set (Bee 2004). These pairwise differences were classified as

199 either within-individual differences (differences between each call and the 19 other calls recorded from

200 the same individual) or among-individual differences (differences between each call from an individual

201 and the 380 calls recorded from the 19 other individuals in the data set). We represent these data as

202 probability density functions of within-individual and among-individual differences for each property

203 (Fig. 2; Fig. S-2, S-3, S-4, S-5).

204 In the context of the dear enemy effect, responding aggressively to a stranger is a "correct

205 detection," whereas mistakenly responding aggressively to a neighbor is a "false alarm." Correctly

206 withholding aggression from a neighbor is a "correct rejection," but mistakenly withholding aggression

207 from a stranger is a "missed detection." For each call property, and over a range of candidate

208 discrimination thresholds (in $0.1 \%$ increments), we computed the probability of false alarm as the

209 proportion of within-individual differences above a candidate threshold and the probability of correct

210 detection as the proportion of among-individual differences above the same candidate threshold. These

211 probabilities were used to create receiver operating characteristic (ROC) curves, which visualize the

212 separation of the probability density functions representing within-individual and among-individual

213 differences by showing the relationship between the probabilities of false alarm and correct detection over

214 the range of possible thresholds. We further quantified this separation by computing the discriminability

215 of among-individual differences as the area between the ROC curve and the positive diagonal of the unit

216 square (Wiley 2006). This measure of discriminability is used in place of the traditional $d^{\prime}$ in signal

217 detection theory, which relies on assumptions of normality and equality of variance. Discriminability

218 varies from 0 , indicating completely overlapping probability density functions, to 0.5 , indicating no

219 overlap between probability density functions. 
The complementary probabilities of false alarm and correct detection are the probabilities of

221 correct rejection and missed detection, respectively. We incorporated the above probabilities in a signal

222 detection theory model of receiver utility (Wiley 1994, 2013b) and found optimal thresholds as those that

223 maximized utility $(U)$, defined as

$U=p_{s}\left(p_{D} d+\left(1-p_{D}\right) m\right)+\left(1-p_{s}\right)\left(p_{F} f+\left(1-p_{F}\right) r\right)$

227 where $p_{s}=$ probability of encountering a "signal" in a window of time (i.e., stranger encounter rate) and 1

$228-p_{s}=$ probability of encountering "noise" (i.e., neighbor encounter rate). Given that a stranger is calling,

$229 p_{D}=$ probability of correct detection (responding aggressively), and $1-p_{D}=$ probability of missed

230 detection (withholding aggression). Given that a neighbor is calling, $p_{F}=$ probability of false alarm

231 (responding aggressively), and $1-p_{F}=$ probability of correct rejection (withholding aggression). This

232 model also includes the payoffs (in terms of relative fitness) of correct and incorrect decisions. The payoff

233 for a correct rejection $(r)$ is the presumed best-case scenario in the context of a dear enemy effect -

234 because it entails correctly ignoring a non-threatening neighbor - and other payoffs are considered

235 relative to it. The payoff for correct detection $(d)$ is lower because a territory owner repels a stranger but

236 may risk injury and energy expenditure if they need to fight the stranger to do so (e.g., Dyson et al. 2013).

237 The payoff for a false alarm $(f)$ is lower still because a neighbor that has been wrongly attacked should be

238 more likely to escalate a fight than a stranger because it has already invested in establishing and

239 defending its territory, whereas the stranger lacks anything to defend (e.g., prior residence effects; (Davies

240 1978; Baugh and Forester 1994; Yang et al. 2020). Finally, the payoff for missed detection ( $m$ ) of a

241 stranger is considered the worst-case scenario because an un-detected stranger could, for example, steal a

242 mating with a female, make use of a defended resource, or take over an entire territory (e.g., relative

243 threat hypothesis; Getty 1987; Temeles 1994; Booksmythe et al. 2010; Siracusa et al. 2017). While we do

244 not have any measurements of these payoffs in nature, we generally expect that in the dear enemy 
context, $r>d>f>>m$. These payoffs influence optimal thresholds based on the ratio of differences

between correct and incorrect responses to neighbors $(r-f)$ and strangers $(d-m)$, that is, $(r-f) /(d-m)$

247 (see Wiley 1994). Given that a neighbor is calling, the difference between the payoffs of correct rejection

248 and false alarm $(r-f)$ is the cost of a false alarm. Given that a stranger is calling, the difference between

249 the payoffs of correct detection and missed detection $(d-m)$ is the cost of a missed detection. Note that

250 by defining optimal thresholds as those that maximize the utility equation above, we are implicitly

251 assuming that encounter rates are fixed and uniform.

We first present predictions based on assumptions that neighbor and stranger encounter rates are

253 equal $\left(p_{s}=0.5\right)$ and the payoff differences between correct and incorrect responses to neighbors and

254 strangers are equal $((r-f) /(d-m)=1)$. This combination of assumptions predicts optimal thresholds

255 equivalent to those predicted by a method that simply maximizes the difference between the probability

256 of correct detection $\left(p_{D}\right)$ and the probability of false alarm $\left(p_{F}\right)$ and, therefore, yields predictions based

257 only on patterns of signal variation. These initial threshold predictions thus serve as a null model of the

258 effect that encounter rates and payoffs have in shifting optimal thresholds; if observed thresholds deviate

259 from our initial predictions, we can then use the observed thresholds to infer how encounter rates and

260 payoffs may have shifted the optimal threshold.

261 We focused our treatment of predicted optimal thresholds on the temporal properties of pulse

262 duration, interval, and period. The predicted optimal thresholds for other call properties can be found in

263 the supplementary material. Additionally, the same acoustic properties measured from different pulses

264 within a call were treated as separate variables when computing pairwise differences, but we pooled these

265 differences together when generating within-individual and among-individual distributions and predicting

266 thresholds because the distributions for different pulses were always similar. Distributions and predicted

267 optimal thresholds for each pulse analyzed separately can be found in the supplementary material.

269 Measuring decision rules 
271 (i) Playback stimuli

272 We used a custom sound synthesis program (written by J. J. Schwartz) to generate synthetic calls (Fig. 1

273 d, e, f) that were modeled after natural calls but that allowed us to systematically manipulate acoustic

274 properties during habituation-discrimination playbacks. Individual pulses consisted of three harmonically

275 related, phase-locked sinusoids with frequencies (and relative amplitudes) of $2669 \mathrm{~Hz}(-30 \mathrm{~dB}), 5338 \mathrm{~Hz}$

$276(0 \mathrm{~dB})$, and $8007 \mathrm{~Hz}(-30 \mathrm{~dB})$, such that $5338 \mathrm{~Hz}$ was the dominant frequency. We edited pulses into calls

277 using Adobe Audition 1.5 (Adobe Systems Inc., San Jose, CA, USA). A call for the habituation stimulus

278 represented the population mean for all properties and consisted of three 36-ms pulses separated by 52-ms

279 intervals, resulting in a pulse period of $88 \mathrm{~ms}$ (Fig. 1f). Each pulse was shaped with 16-ms exponential

280 convex onset and offset ramps that reached 50\% maximum amplitude at $4 \mathrm{~ms}$ after onset and before

281 offset, respectively. We created a 1-minute-long stimulus consisting of 23 calls at a rate of 1 call/2.5 s.

282 We appended two repetitions of this stimulus followed by two minutes of silence to create a 4-minute

283 stimulus period (Fig. 1d). These stimuli were saved as WAV-files (44.1 kHz sampling rate, 16-bit

284 resolution). During the discrimination phase of the experiment, we presented a novel stimulus that was

285 designed to simulate a different individual based on differences in the temporal properties of pulses. To

286 estimate a behavioral threshold for responding aggressively following habituation, we tested four novel

287 stimuli that featured a $3 \%, 6 \%, 9 \%$, or $12 \%$ change in pulse temporal properties. These values were

288 selected to span the range of optimal discrimination thresholds predicted by our signal detection theory

289 model under the assumptions of equal encounter rates and payoffs for interactions with neighbors and

290 strangers (see below). These manipulations involved changing three inter-related temporal properties of

291 pulses (pulse duration + pulse interval = pulse period) simultaneously and by the same percentage

292 difference to account for their natural intercorrelation. All other acoustic properties were held constant

293 between stimuli used during the habituation and discrimination phases. For each experimental treatment,

294 half of the males heard an increase in pulse temporal properties, and half heard a decrease. As a control,

295 we also included a $0 \%$ change treatment, which consisted of additional broadcasts of the habituation 
296 stimulus during the discrimination phase. Stimuli for all five treatments had the same pulse duty cycle

297 (pulse duration/pulse period) of 0.41.

299 (ii) Playback protocol.

300 We performed 62 habituation-discrimination playback tests with 17 male subjects. This species is

301 sexually dichromatic, and males are easily identified in the field (Engelbrecht-Wiggans and Tumulty

302 2019). Additionally, males were photographed so that they could be individually identified based on

303 individually distinctive dorsal patterns. All playbacks began in the morning (0700 - 1100 hours), when

304 this species is most active. Stimuli were played from a digital audio player (iPod, Apple, Cupertino, CA,

305 USA) connected to an amplified field speaker (Saul Mineroff Electronics, Elmont, NY, USA). The

306 speaker was calibrated to produce stimuli at $80 \mathrm{~dB}$ SPL measured at $1 \mathrm{~m}$, which is within the range of

307 variation in call amplitude for this species (Pettitt et al. 2012). To begin a test, we chose a calling male

308 and positioned the speaker on a tripod $1.5-2 \mathrm{~m}$ from the subject in a direction with no adjacent

309 neighbors. The speaker was positioned at an equivalent height to the subject and so that it contacted a

310 bromeliad leaf to simulate a realistic calling location. We also set up a microphone (Sennheiser ME-66,

311 Wedemark, Germany) and digital recorder (Marantz PMD-620, Kanagawa, Japan; 44.1kHz sampling rate,

312 16-bit resolution) to record the subject's vocal responses. Subjects sometimes stopped calling during

313 speaker set-up, so we waited at least 5 minutes or until it resumed calling to begin the playback.

314 We started a test with a 4-minute pre-stimulus period, during which we observed the subject's

315 behavior in the absence of any stimulus. We then began the habituation phase, during which we

316 repeatedly played the 4-min habituation stimulus (Fig. 1d). This phase of the test simulated the arrival of

317 a new neighbor. During each minute of the test, we recorded four behavioral measures of aggression: (1)

318 the number of aggressive calls, (2) the number of pseudo-aggressive calls, (3) the subject's approach

319 distance, and (4) the subject's closest position to the speaker. Golden rocket frogs have distinct aggressive

320 calls (Bourne et al. 2001; Pettitt et al. 2012), which consist of long trains of pulses typically preceded by

321 several introductory pulses with relatively longer inter-pulse intervals. We classified all calls with at least 
seven pulses as aggressive calls. Calls consisting of the characteristic introductory pulses without a

subsequent train of pulses were scored as "pseudo-aggressive calls" (Tumulty et al. 2021). Call counts

were always confirmed from the audio recordings of each test. We also noted the subject's position at the start of each minute of the test as well as its closest position to the speaker during each minute. After the test, we measured the distances from the speaker to each noted position, and from these measurements, we computed the subject's approach distance (net displacement towards the speaker) during each minute as well as the distance from the speaker to the subject at its closest position to the speaker during each minute. We also recorded air temperature at the final location of the frog. The range in temperatures during our playbacks was 22.4 to $26.8^{\circ} \mathrm{C}\left(\right.$ mean $\left.=24.3^{\circ} \mathrm{C}\right)$.

We aborted the test if the subject did not show any aggressive behaviors during the first three stimulus periods of the habituation phase (i.e., $12 \mathrm{~min}$ ), started interacting aggressively with a real male, or started courting a female. We played the habituation stimulus for at least six stimulus periods (i.e., 24 mins) and until the subject met our habituation criteria of no aggressive calls, pseudo-aggressive calls, or approach movements towards the speaker for three consecutive stimulus periods (maximum habituation time was $5.6 \mathrm{hrs}$ ). Once these habituation criteria were met, we switched to the designated discrimination stimulus (i.e., a $0 \%$ [control], $3 \%, 6 \%, 9 \%$, or $12 \%$ change) for three stimulus periods during the discrimination phase. After the test, we caught the male, if possible, to confirm its identity. While we did not always succeed in catching subject males after tests, their territories are remarkably stable (Tumulty and Bee 2021), and every time we caught a male it was the same individual as assumed based on location.

341 We attempted to test each subject in all five treatments; however, some tests were unsuccessful for a

342 variety of reasons (see above), so not all 17 subjects were tested in all treatments. We allowed at least five

343 days between consecutive tests to minimize the potential effects of long-term habituation to the

344 habituation stimulus. There was no effect of number of attempted playback tests on a subject's likelihood

345 of response (logistic mixed effects model: $\chi^{2}=2.4, p=0.12, n=118$ attempted tests), nor was there an

346 effect on their time to reach habituation criteria (linear mixed effects model: $\chi^{2}=0.2, p=0.65, n=62$

347 successful tests), one measure of overall aggressiveness. The final sample sizes for each treatment (and 
sample sizes for the corresponding increases and decreases in pulse properties) were as follows: $0 \%, n=$

$12 ; 3 \%, n=12$ (6 increased and 6 decreased, respectively); $6 \%, n=12$ (6 and 6); 9\%, $n=13$ (6 and 7);

and $12 \%, n=13$ (6 and 7). Treatment order was randomly assigned to each subject, and we changed the

351 file names of the stimuli so that observers were blind to treatments when conducting playbacks and

352 scoring aggressive behaviors.

354 (iii) Statistical analyses.

355 To examine the effects of treatments on aggressive responses, we summarized data by block, which

356 consisted of three consecutive stimulus periods. For each block, we summed the number of aggressive

357 and pseudo-aggressive calls and summed the approach distances. To control for slight variation between

358 tests in initial speaker distance, we expressed the subject's closest position to the speaker as a proportion

359 of the total distance between the speaker and subject at the start of the test; we used the inverse of this

360 proportion so that higher values indicate a closer approach to the speaker. We subjected these four

361 correlated measures of aggression to a principal components transformation (centered to zero and scaled

362 to unit variance) using the 'prcomp' function in R (R Core Team 2017) and used the first principal

363 component as an aggression index. This aggression index explained $56 \%$ of the overall variation in

364 aggressive behaviors and was positively correlated with the number of aggressive calls (loading $=0.52$ )

365 and pseudo-aggressive calls (0.40), approach distance (0.57), and closest position to the speaker (0.50).

366 Additionally, results for each measure of aggression are plotted separately in the supplementary material

367 (Fig. S-6, S-7, S-8, S-9). To estimate a behavioral threshold for behaviorally discriminating between

368 neighbors and strangers, we tested for a change in aggression index between the last block of the

369 habituation phase and the single block of the discrimination phase using Wilcoxon signed rank tests

370 within each treatment. For these tests we report $V$ statistics, which correspond to the sum of positive

371 ranks. We estimated the behavioral threshold as occurring in the range between the smallest percentage

372 change in the novel stimulus that elicited significant recovery of aggression and the largest change that

373 did not. 
Modeling encounter rates and payoffs

376 After estimating the threshold for behaviorally discriminating between neighbors and strangers in the

377 playback tests, we returned to our signal detection theory model and explored the ranges of parameter

378 values for stranger encounter rates and relative payoffs that could result in the observed behavioral

379 threshold. Because classic methods for determining parameters based on measured thresholds rely on

380 assumptions of normality and equal variance (Gescheider 1976), which our data did not meet, we instead

381 determined parameter values by computing optimal thresholds from the receiver utility equation for a

382 large range of possible values of stranger encounter rates and relative payoffs. We varied stranger

383 encounter rates $\left(p_{S}\right)$ from 0 to 1 , in 0.1 increments. To vary relative payoffs $((r-f) /(d-m))$, we varied

384 values of $\mathrm{r}$ and $\mathrm{d}$ such that the resulting numerator and denominator of this ratio each varied from 1 to 20 ,

385 allowing us to explore parameter space ranging from equivalent costs of false alarms $(r-f)$ and missed

386 detections $(d-m)$ (i.e., $(r-f) /(d-m)=1)$ to scenarios in which the costs of false alarms are twenty times

387 greater than the costs of missed detections $((r-f) /(d-m)=20)$ and scenarios in which the costs of missed

388 detections are twenty times greater than the costs of false alarms $((r-f) /(d-m)=0.05)$. We then selected

389 the combinations of these parameters that resulted in the optimal thresholds estimated from our playback

390 tests.

392 Results

\section{Predicted optimal decision rules}

395 As illustrated in Figure 2a (see also Fig. S-2, S-3, S-4, S-5), there was overlap in the distributions of

396 within-individual and among-individual differences in acoustic properties, thus demonstrating that these

397 properties vary continuously within the population. Importantly, however, among-individual differences

398 were larger than within-individual differences for all acoustic properties. Compared with other properties,

399 the temporal properties of pulses (pulse duration, pulse interval, and pulse period) and spectral properties 
400 had larger separations between distributions representing within-individual differences and among-

401 individual differences (Table S-1, S-3; Fig. 2a; Fig. S-2, S-3, S-4, S-5). This separation is visualized in

402 the ROC curves in Figure 2b, which are greatly offset from the diagonal. Pulse period had a slightly larger

403 value of discriminability (0.28) than pulse duration (0.24) and pulse interval (0.23) (Fig. 2b, Table S-1, S-

404 3). Most within-individual differences in pulse duration, pulse interval, and pulse period were less than

$4055 \%$ (Fig. 2a). While small among-individual differences $(<5 \%)$ in these three properties were not

406 uncommon (constituting $25 \%$ to $36 \%$ of comparisons), most among-individual differences in pulse

407 duration, pulse interval, and pulse period were above 5\% (Fig. 2a). Assuming equivalent relative payoffs

$408((r-f) /(d-m)=1)$ and equal neighbor and stranger encounter rates $\left(p_{s}=1-p_{s}=0.5\right)$, the predicted

409 optimal thresholds for discriminating between individuals based on the temporal properties of pulses

410 ranged between 5\% and 10\%: for pulse duration and pulse period the predicted optimal thresholds were

$4115.1 \%$ and $6.6 \%$, respectively, while that for pulse interval was somewhat higher at 9.2\% (Fig. 2a, Table S-

412 1). The distributions of within-individual and among-individual differences and predicted thresholds

413 using data pooled across pulses (Fig. 2a, Table S-1) were very similar to those for each pulse analyzed

414 separately (Table S-1, S-3, Fig. S-2, S-3, S-4).

415 
(a)
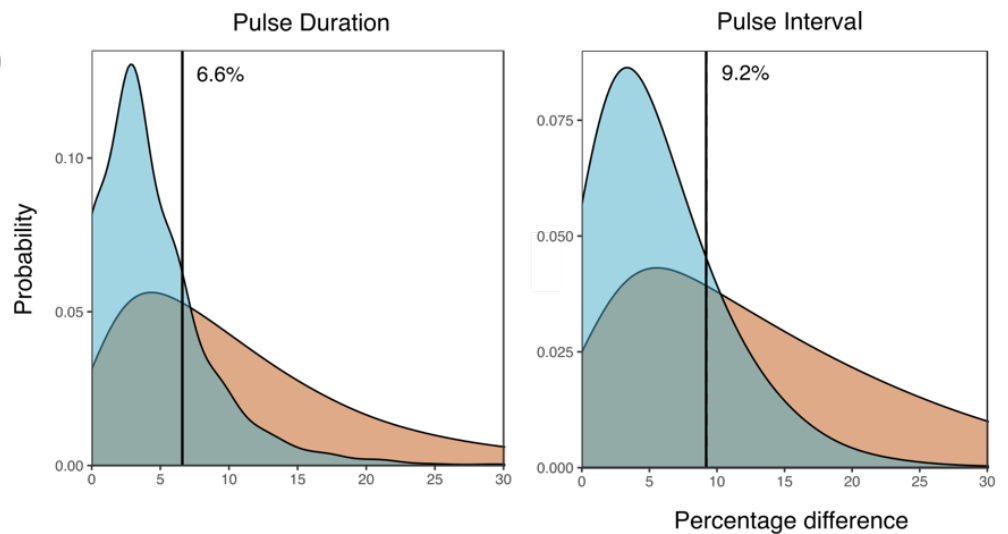

(b)
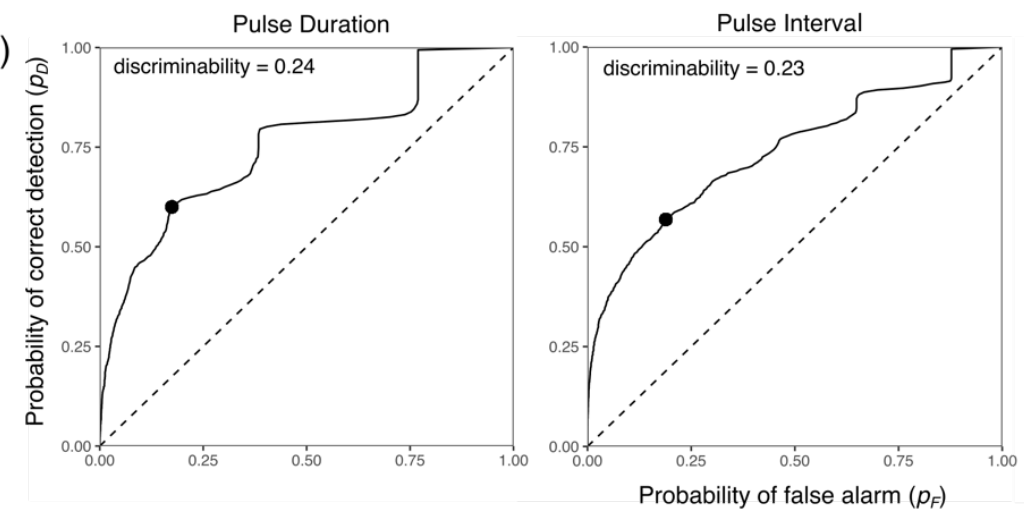

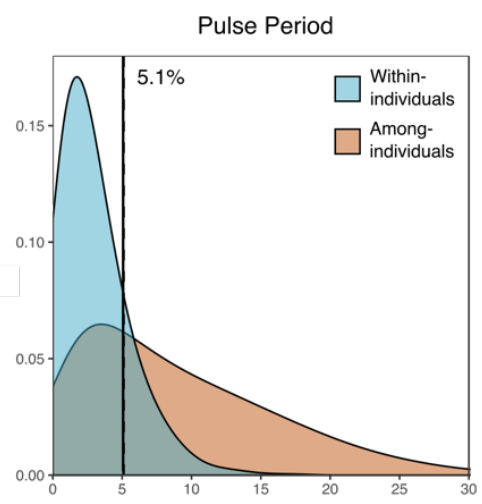

Figure 2. (a) Probability density functions of the within-individual and among-individual percentage

418 differences in pulse duration, pulse interval, and pulse period. Data are pooled from all three pulses in a

419 call. The vertical line in each graph represents the predicted optimal discrimination threshold for that call

420 property based on signal variation, assuming equivalent neighbor and stranger encounter rates (i.e., $p_{s}=$

$4210.5)$ and relative payoffs (i.e., $(r-f) /(d-m)=1)$ ). Optimal thresholds, computed as the threshold that maximizes receiver utility, represent the percentage change in each property that was predicted to elicit behavioral discrimination from territorial males in field playback tests. (b) Receiver operating

424 characteristic (ROC) curves generated from within-individual and among-individual differences in each 425 acoustic property. These curves are generated by computing the probability of correct detection $\left(p_{D}\right)$ and 426 probability of false alarm $\left(p_{F}\right)$ for each possible threshold. Points show the location of $p_{D}$ and $p_{F}$ that

427 correspond to the predicted optimal thresholds in (a). Discriminability values were computed as the area 428 between the ROC curve and the positive diagonal of the unit square (dotted line). 


\section{Measured decision rules}

431 Territorial males responded to synthetic call playbacks with aggressive behaviors that were typical of

432 responses to playbacks of natural calls (Tumulty and Bee 2021) as well as natural aggressive interactions

433 between males (personal observations). Males often approached the speaker while the stimulus was

434 playing and retreated to the centers of their bromeliads, away from the speaker, during intervening

435 periods of silence. Some males approached all the way to the speaker, apparently searching for the

436 simulated intruder. Males also responded to playbacks with aggressive calls, which were typically

437 produced during the periods of silence between stimuli. These aggressive responses gradually decreased

438 with repeated broadcasts of the stimulus during the habituation phase (Fig. 3), but the amount of time it

439 took for subjects to meet habituation criteria varied considerably among individuals. The median amount

440 of time to reach these criteria was $1 \mathrm{hr}$ (or 15 stimulus periods) and ranged from 24 min (6 stimulus

441 periods) to $5.6 \mathrm{hrs}$ (84 stimulus periods).

The threshold for behaviorally discriminating between the calls of different simulated individuals

443 based on the percentage differences in pulse temporal properties was above $9 \%$ but below $12 \%$. Only a

$44412 \%$ change in the temporal properties of pulses elicited an obvious and statistically significant recovery

445 of aggression during the discrimination phase $(V=44, p=0.013, n=13$; Fig. 4). This treatment

446 corresponds approximately to a 10-ms change in pulse period, a 4-ms change in pulse duration, and a 6-

447 ms change in pulse interval. In this treatment group, there was no difference in recovery of aggression

448 between subjects that experienced an increase versus a decrease in the values of pulse temporal properties

449 (Wilcoxon rank sum test, $\mathrm{W}=18.5, p=0.77 ; n_{1}=6, n_{2}=7$ ), indicating that subjects were responding to

450 the change in these properties instead of revealing a bias for responding with more or less aggression to

451 longer or shorter pulse temporal properties. The median difference in the aggression index between the

452 last block of the habituation phase and the block of the discrimination phase was zero for the $0 \%, 3 \%$,

$4536 \%$, and $9 \%$ treatments (Fig. 4b). A 9\% change elicited recovery of aggression in some subjects (Fig. 4)

454 during the discrimination phase, but the difference from the last block of the habituation phase was not 
455 statistically significant ( $V=18, p=0.14, n=13$; Fig. 4). Similarly, there were no statistically significant

456 differences in aggression between the last block of the habituation phase and the block of the

457 discrimination phase for the $0 \%$ change control $(V=9, p=0.20, n=12), 3 \%$ change $(V=16, p=0.29, n$

$458=12)$, or $6 \%$ change $(V=33, p=0.61, n=12)$ treatments (Fig. 4). An examination of the separate

459 components of the aggression index suggest significant recovery of aggression in the $12 \%$ treatment was

460 driven primarily by renewed physical approaches towards the playback speaker (Figs. S-8, S-9) and not

461 renewed aggressive calling (Figs. S-6, S-7).

462

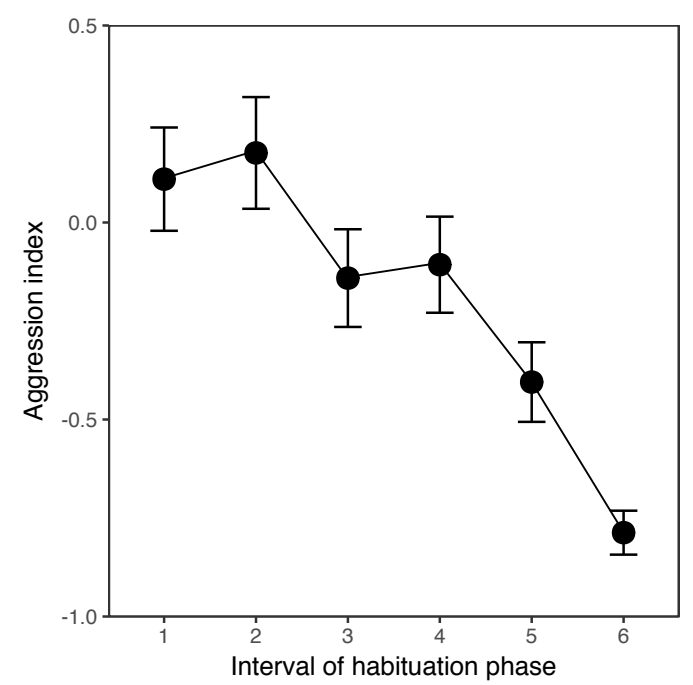

464 Figure 3. Mean $( \pm \mathrm{SE})$ aggression index of territorial male golden rocket frogs to synthetic advertisement

465 call playbacks over the course of the habituation phase ( $n=62$ tests of 17 males). Because subjects took

466 different amounts of time to reach habituation criteria, here we visualized trends in aggression during the

467 habituation phase by partitioning this phase into 6 intervals ( 6 being the minimum number of stimulus

468 periods), each constituting $16.7 \%$ of the total time a subject required to reach the criteria. To do this we

469 first summarized data in the same manner as described in the methods but by stimulus period. We then

470 subjected these data to a principal components transformation to compute an aggression index and

471 calculated the mean aggression index during each interval across subjects. 


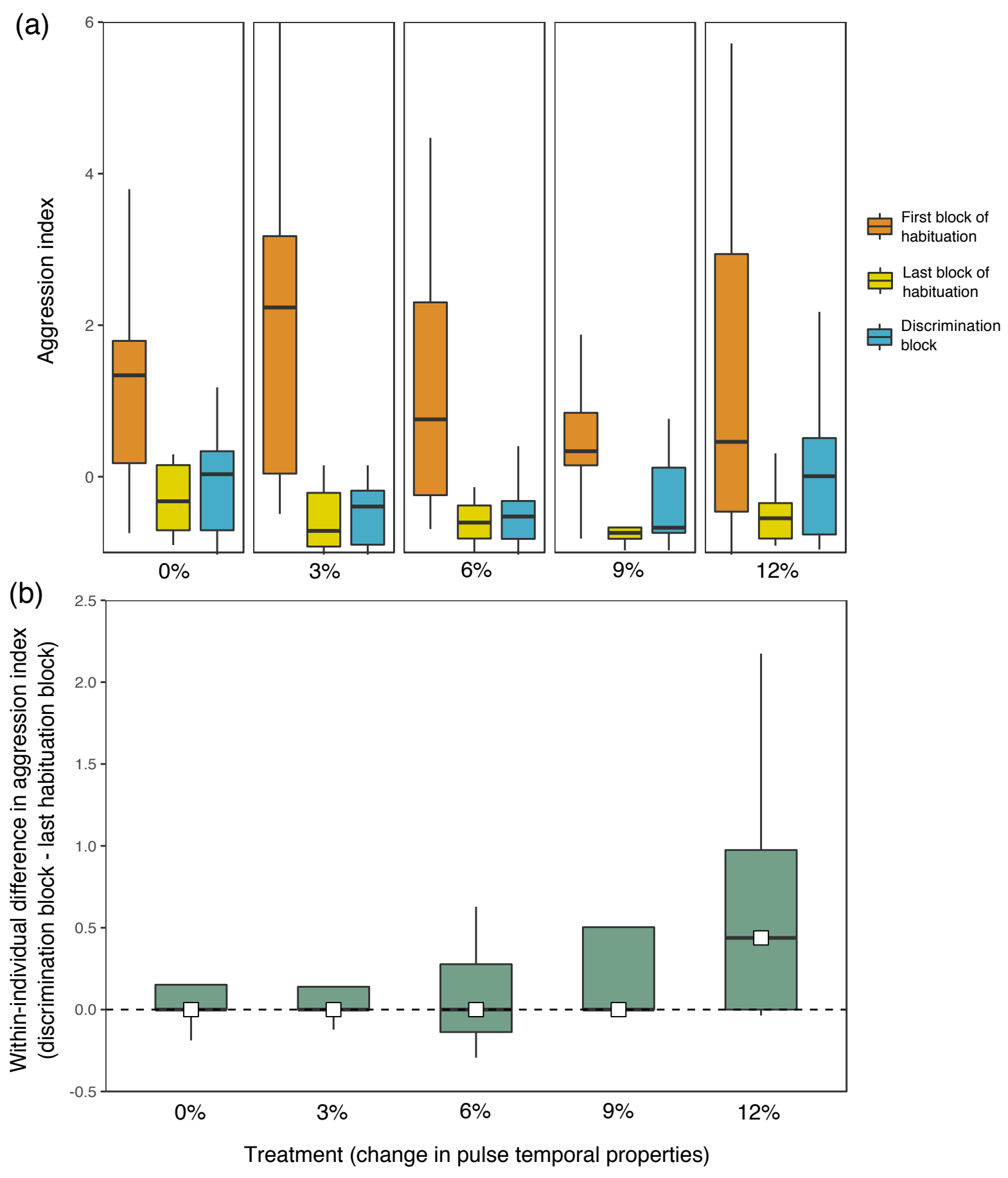

474 Figure 4. (a) Boxplots of the aggression index of territorial male subjects during the first and last blocks

475 of the habituation phase and during the block of the discrimination phase for each treatment $(0 \%, n=12$;

$4763 \%, n=12 ; 6 \% n=12 ; 9 \% n=13 ; 12 \%, n=13$ ). (b) Boxplots showing the within-subjects difference in

477 aggression index between the last block of the habituation phase and the discrimination phase for each 
treatment. Horizontal bars represent the median, box hinges represent the interquartile range, and whiskers extend to the range but no further than 1.5 times the interquartile range. White squares also show the median in (b) to aid visualization.

\section{Modeling encounter rates and payoffs}

483 Exploring the range of parameters that could have produced the observed behavioral threshold of 9-12\%

484 revealed some differences from our starting assumptions of equal neighbor and stranger encounter rates

485 (i.e., $\left.p_{S}=1-p_{S}=0.5\right)$ and equivalent relative payoffs $((r-f) /(d-m)=1)$ that produced our initial prediction of an optimal discrimination threshold between $5 \%$ and $10 \%$. The range of possible parameter values that could have resulted in the observed threshold of $9-12 \%$ did not overlap with our initial assumptions for pulse duration and pulse period but did overlap with those assumptions for pulse interval (Fig. 5). For pulse duration and pulse period, at equivalent relative payoffs, the observed threshold could have been produced by lower stranger encounter rates. Alternatively, if stranger encounter rates are kept

491 at 0.5 for these properties, the observe thresholds could have been produced by increased costs of false

492 alarms (mistakenly attacking a neighbor) (Fig. 5). For all three properties, the observed thresholds are also 493 consistent with ecologically realistic scenarios in which stranger encounter rates are rare and the costs of 494 missed detections of strangers are greater than the costs of false alarms (lower left quadrants of Fig. 5).

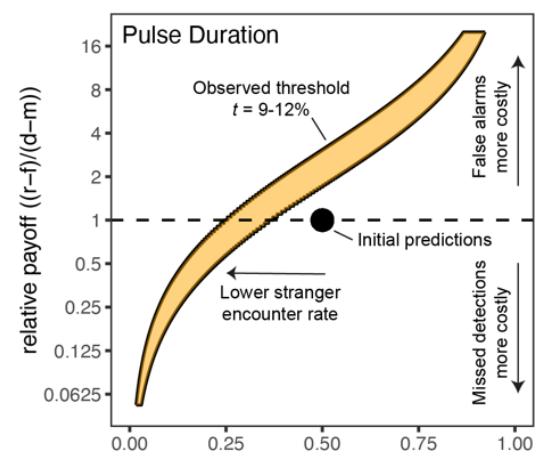

496
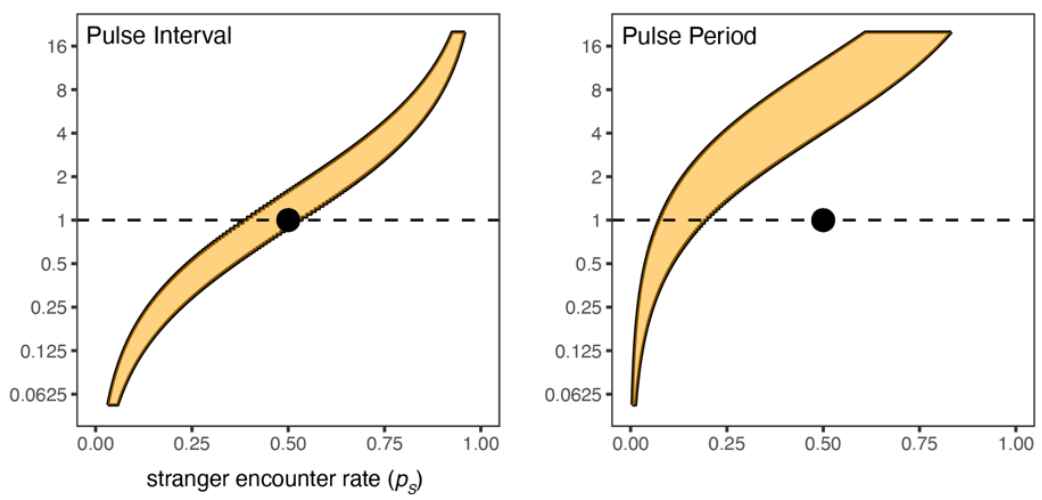
497 Figure 5. Parameter space (orange curve) for the joint values of stranger encounter rates $\left(p_{S}\right)$ and relative 498 payoffs $((\mathrm{r}-\mathrm{f}) /(\mathrm{d}-\mathrm{m}))$ that could result in the observed behavioral discrimination threshold of between $9 \%$

499 and $12 \%$ changes in pulse temporal properties. The point in each plot displays the initial assumptions of

500 equal encounter rates to neighbors and strangers $\left(p_{S}=0.5\right)$ and equivalent costs of false alarms and missed 501 detections $((\mathrm{r}-\mathrm{f}) /(\mathrm{d}-\mathrm{m})=1)$.

\section{Discussion}

In this study, we addressed two major questions about social recognition: what are the decision

505 rules used to discriminate among individuals, and how are these rules shaped by patterns of individual

506 variation in signals, the rates of encountering different individuals, and the payoffs associated with

507 interactions with different individuals? Similar to responses to repeated playbacks of natural calls

508 (Tumulty et al. 2021), repeated exposures to synthetic calls simulating a new neighbor led to both

509 habituation of territorial aggression and the formation of a memory of individually distinctive signal

510 properties. Males employed a threshold for behaviorally discriminating individual differences in calls that

511 required a difference in pulse temporal properties between $9 \%$ and $12 \%$ : changes from the habituation

512 stimulus of $9 \%$ or less failed to elicit recovery of aggression while a $12 \%$ change elicited renewed

513 territorial aggression. This empirically determined range for a threshold partially overlaps, but extends

514 somewhat higher than, the predicted range of $5 \%$ to $10 \%$ based on our initial signal detection theory

515 model. Notably, however, our initial predictions were based on patterns of signal variation alone, as they

516 assumed both that neighbors and strangers were encountered at equivalent rates and that the costs of false

517 alarms (incorrectly attacking a neighbor) and missed detections (incorrectly ignoring a stranger) were

518 equivalent. We interpret the relatively close correspondence between observed and predicted thresholds

519 as evidence that patterns of individual variation in signals have been an important factor shaping the

520 evolution of behavioral decision rules in the golden rocket frog's recognition system. Nevertheless,

521 exploring the parameter space of the model that produced thresholds in the range of $9-12 \%$ showed that

522 both lower rates of encountering strangers than neighbors and differences in the costs of false alarms and 
523 missed detections could improve the correspondence between observed and predicted thresholds.

524 Understanding the importance of these two additional factors in broader ecological and evolutionary

525 contexts first requires consideration of likely encounter rates and costs of errors in natural settings.

526 The assumption that strangers and neighbors are encountered at equal rates is almost certainly not

527 true for most territorial animals. Male golden rocket frogs, for example, typically call from their spatially

528 clumped territories every day, often for several hours each day (Bourne et al. 2001; Pettitt et al. 2012,

529 2020; Tumulty and Bee 2021). Because of this, territorial males likely hear the calls of their nearby

530 neighbors more frequently than they hear the calls of strangers and thus risk paying the costs of false

531 alarms (incorrectly attacking a neighbor) more frequently than the costs of missed detection (incorrectly

532 ignoring a stranger). Male golden rocket frogs may have evolved somewhat higher discrimination

533 thresholds than predicted based on signal variation alone to decrease the rate at which they pay the costs

534 of false alarms (Fig. 5). Of course, the relative payoffs themselves also impact optimal thresholds in

535 addition to the rates at which different payoffs are incurred. Our initial predictions assumed that the

536 payoff differences of correct and incorrect responses to neighbors and strangers were equal, an

537 assumption that may not hold in many cases. The model showed that, at equal encounter rates, our

538 observed threshold could have been produced by the costs of false alarms being greater than the costs of

539 missed detections. Relatively high costs of false alarms could be the case if neighbors are particularly

540 likely to escalate fights when challenged, even with established "dear enemies". This scenario would be

541 consistent with prior-residence effects in which territory holders are more likely to escalate and win

542 contests over intruders (Davies 1978). Prior residence effects are well documented among territorial

543 animals (Kokko et al. 2006), including in another poison frog (Baugh and Forester 1994; Yang et al.

544 2020). In the context of the dear enemy effect, however, the costs of missed detections may ultimately be

545 greater than the costs of false alarms. Strangers are generally thought to pose a greater threat to territory

546 owners than neighbors, because, unlike neighbors who already have territories of their own, strangers

547 represent individuals who may attempt to take over another male's defended resources (Getty 1987;

548 Temeles 1994). A false alarm may result in a brief fight with a neighbor, which would be worse than 
correctly ignoring that neighbor. But the difference in payoffs between correct and incorrect responses to

strangers may be greater because strangers moving through a territory network that are quickly detected

can be driven off with an aggressive display, but strangers that are un-detected could potentially steal a

mating with a female, make use of defended reproductive resources, or possibly usurp an entire territory.

553 Even a stranger establishing a new territory within an existing territory network represents the addition of

554 another nearby competitor for limited resources (Getty 1987). Relatively higher costs of missed detections

555 than false alarms decrease the predicted optimal threshold. Overall, while we presently lack the necessary

556 data to make reliable estimates for encounter rate and payoff parameters, the signal detection theory

557 model fits the observed threshold under ecologically realistic conditions of low stranger encounter rates,

558 even when failing to respond to a stranger (missed detection) is more costly than mistakenly attacking a

559 neighbor (false alarm) (Fig. 5, lower left quadrant). Our approach of parameterizing our signal detection

560 theory model with measures of signal variation was useful for predicting receiver decision rules in the

561 dear enemy context and for interpreting how encounter rates and payoffs may have shaped these decision

562 rules, either over evolutionary time or perhaps as a result of behavioral plasticity in social environments

563 where encounter rates and costs of errors fluctuate (e.g., Stoddard 1996). We think this approach, as well

564 as signal detection theory more broadly, will also yield useful insight when applied to other contexts of

565 animal communication (Wiley 1994, 2006, 2013b; Scharf et al. 2020; Sumner and Sumner 2020).

567 individually distinctive acoustic cues for discriminating between individuals. While acoustic and

568 statistical analyses typically support the widely held view that animal vocalizations possess individually

569 distinctive properties (Bee et al. 2016), surprisingly few previous studies have identified specific signal

570 properties used by receivers to discriminate among individuals. Studies of the perceptual basis of the dear

571 enemy effect in white-throated sparrows (Brooks and Falls 1975b), field sparrows (Nelson 1989) and

572 North American bullfrogs (Bee and Gerhardt 2001a, 2001b, 2001c, 2002) have identified "pitch" (i.e.,

573 acoustic frequency) as an individually distinctive property of vocalizations that can be used to

574 discriminate between neighbors and strangers. Our finding that male golden rocket frogs can learn to 
575 discriminate individual differences in the temporal properties of vocalizations reveals a new perceptual

576 mechanism underlying the dear enemy effect. Across animal groups, temporal patterns in acoustic signals

577 convey biologically important information (e.g., Pollack and Hoy 1979; Stratton and Uetz 1983; Illes et

578 al. 2006). Among frogs, temporal information plays key roles in species recognition (Gerhardt 2001;

579 Vélez et al. 2012), mate choice (Gerhardt et al. 1996; Akre and Ryan 2010; Tanner et al. 2017), and male-

580 male competition (Wells and Schwartz 1984; Gerhardt et al. 2000; Burmeister et al. 2002). Our study

581 extends the role of temporal information in frog communication to social recognition. In golden rocket

582 frogs, the temporal properties of pulses within calls are the most individually distinctive properties of

583 advertisement calls (Pettitt et al. 2013), and males in our experiment learned to recognize pulse temporal

584 properties of the calls of simulated new neighbors, discriminating between calls that differed by $12 \%$ in

585 these properties. The pattern of habituation and discrimination we observed in our experiment would

586 allow territorial males to ignore neighbors and respond aggressively to a large subset of strangers, thereby

587 producing a dear enemy effect based solely on patterns of individual variation in pulse temporal

588 properties. Whether males also use other acoustic properties of calls to recognize neighbors remains to be 589 investigated.

The magnitude of change in pulse temporal properties that was discriminated by male golden

591 rocket frogs is noteworthy and deserves additional comment. The percentage change of $12 \%$ in the

592 discrimination phase corresponded to absolute changes of approximately $10 \mathrm{~ms}$ in pulse period, $4 \mathrm{~ms}$ in

593 pulse duration, and $6 \mathrm{~ms}$ in pulse interval. Similar sensitivity of the anuran auditory system to such small

594 differences in temporal properties has been demonstrated previously, but only in studies of unlearned

595 behaviors related to species recognition and mate choice conducted under highly controlled laboratory

596 conditions (Gerhardt and Schul 1999; Bush et al. 2002). Neurophysiological studies indicate that such

597 temporal discrimination likely involves neurons in the anuran midbrain that are tuned to particular pulse

598 durations and intervals (Edwards et al. 2002; Rose 2014). However, that a frog can discriminate small

599 temporal differences in acoustic signals on the order of $4 \mathrm{~ms}$ to $10 \mathrm{~ms}$ under natural conditions based on

600 learning, as we have shown, is unprecedented in the literature and deserves additional study. At present, 
601 for example, it remains to be determined whether golden rocket frogs perceive individual differences in

602 vocalizations categorically (Tanner and Tumulty 2020), similar to the categorical perception in songbirds

603 that underlies discrimination between types of vocalizations (Nelson and Marler 1989) or beak colors

604 (Caves et al. 2018). It will also be important to discover whether frogs can perceptually discriminate

605 between smaller differences than they behaviorally discriminated in our experiment, that is, whether their

606 just-noticeable differences are smaller than their just-meaningful differences.

607 We emphasize that there are many aspects of territory establishment and interactions with

608 neighbors that were not captured in our playback experiment. Territory disputes, for example, often

609 escalate to intense bouts of physical aggression in diverse animal species (e.g., fiddler crabs (Booksmythe

610 et al. 2010), chameleons (Ligon 2014), cichlid fish (O’Connor et al. 2015), songbirds (Krebs 1982), and

611 chimpanzees (Wrangham 1999)). Such interactions are also common among territorial frogs (Dyson et al.

612 2013), and in golden rocket frogs, physical aggression involves wrestling and chasing that can sometimes

613 span several days (personal observations). Such physical and visual interactions create the potential for

614 territory holders to acquire information about the reliability and fighting ability of neighbors and associate

615 that information with memories of their neighbors' communication signals. While these interactions are

616 certainly important in real dear enemy relationships, the habituation-discrimination experiment

617 nevertheless allowed us to experimentally probe the decision rules used to socially categorize variation in

618 calls. As a taxonomically widespread form of non-associative learning, habituation likely plays a key role

619 allowing territory holders to learn about their neighbors and adjust their aggression accordingly

620 (Shettleworth 2010), as demonstrated in previous studies of fish (Peeke and Peeke 1973; Peeke 1984),

621 frogs (Owen and Perrill 1998), and songbirds (Petrinovich 1984). Previous studies in other frogs, for

622 example, have shown repeated exposure to conspecific calls can lead to habituation of aggression that can

623 enable neighbor recognition and the dear enemy effect (Bee and Gerhardt 2001b, 2001c, 2002) or simply

624 allow males to modulate their aggression in a way that allows them to track changes in the local density

625 of conspecific males in the chorus (Brenowitz and Rose 1994; Marshall et al. 2003; Reichert 2010). We

626 have recently shown that the specificity of social categories that are learned through habituation has been 
627 a key target of selection enabling a dear enemy effect in golden rocket frogs (Tumulty et al. 2021). A

628 closely related species that does not discriminate between neighbors and strangers (Kai rocket frogs, $A$.

629 kaiei) exhibits similar patterns of habituation to conspecific calls, but unlike golden rocket frogs, Kai

630 rocket frogs show habituation that is generalized to conspecific calls as they do not discriminate

631 individual differences in calls (Tumulty et al. 2021). Thus, it appears that key parameters of habituation,

632 such as stimulus specificity, are evolutionarily labile in frogs and have been shaped by natural selection to

633 allow individuals to navigate their social environment.

The abilities of animals to categorize continuous variation in acoustic signals underlies the

635 recognition of mates (Gerhardt 2001; Baugh et al. 2008), rivals (Nelson 1989; Nelson and Marler 1990;

636 Amézquita et al. 2011), and offspring (Ehret and Haack 1981; Ehret 1992), as well as familiar individuals

637 in the context of social recognition (Nelson 1989; Bee and Gerhardt 2001b; this study). Key to such

638 categorizations is the use of decision rules that have been shaped by natural selection. In the context of

639 social recognition based on communication signals, these decision rules should reflect not only the

640 perceptual resolution of a receiver's sensory system, but also the patterns of individual variation in

641 signals, the payoffs of correct and incorrect decisions, and the rates at which animals are likely to incur

642 these payoffs (Reeve 1989; Wiley 1994). As highlighted by the present study, signal detection theory

643 provides a useful framework for integrating signal analyses, models of receiver utility that consider

644 encounter rates and payoffs, and empirical studies of behavioral discrimination thresholds to better

645 understand both the mechanisms and evolution of decision rules for social recognition. 


\section{References}

647 Akre, K. L., and M. J. Ryan. 2010. Complexity increases working memory for mating signals. Current

648 Biology 20:502-505.

649 Amézquita, A., S. V. Flechas, A. P. Lima, H. Gasser, and W. Hödl. 2011. Acoustic interference and

650 recognition space within a complex assemblage of dendrobatid frogs. Proceedings of the National

651 Academy of Sciences 108:17058-63.

652 Aubin, T., and P. Jouventin. 2002. How to vocally identify kin in a crowd: the penguin model. Advances

653 in the Study of Behavior 31:243-277.

654 Baugh, A. T., K. L. Akre, and M. J. Ryan. 2008. Categorical perception of a natural, multivariate signal:

655 mating call recognition in túngara frogs. Proceedings of the National Academy of Sciences of the United

656 States of America 105:8985-8988.

657 Baugh, J. R., and D. C. Forester. 1994. Prior residence effect in the dart-poison frog, Dendrobates

658 pumilio. Behaviour 131:207-224.

659 Bee, M. A. 2004. Within-individual variation in bullfrog vocalizations: Implications for a vocally

660 mediated social recognition system. The Journal of the Acoustical Society of America 116:3770.

661 Bee, M. A., and H. C. Gerhardt. 2001a. Neighbour-stranger discrimination by territorial male bullfrogs

662 (Rana catesbeiana): I. Acoustic basis. Animal Behaviour 62:1129-1140.

$663 \longrightarrow$ 2001b. Neighbour-stranger discrimination by territorial male bullfrogs (Rana catesbeiana): II.

664 Perceptual basis. Animal Behaviour 62:1141-1150.

665 2001c. Habituation as a mechanism of reduced aggression between neighboring territorial male

666 bullfrogs (Rana catesbeiana). Journal of Comparative Psychology 115:68-82.

667 2002. Individual voice recognition in a territorial frog (Rana catesbeiana). Proceedings of the

668 Royal Society of London B: Biological Sciences 269:1443-1448.

669 Bee, M. A., M. S. Reichert, and J. Tumulty. 2016. Assessment and recognition of competitive rivals in

670 anuran amphibians. Advances in the Study of Behavior 48:161-249.

671 Beecher, M. D. 1989. Signalling systems for individual recognition: an information theory approach. 
Animal Behaviour 38:248-261.

673 Booksmythe, I., M. D. Jennions, and P. R. Y. Backwell. 2010. Investigating the 'dear enemy'

674 phenomenon in the territory defence of the fiddler crab, Uca mjoebergi. Animal Behaviour 79:419-423.

675 Bourne, G. R., A. Collins, A. Holder, and C. McCarthy. 2001. Vocal communication and reproductive

676 behavior of the frog Colostethus beebei in Guyana. Journal of Herpetology 35:272-281.

677 Brenowitz, E. A., and G. J. Rose. 1994. Behavioural plasticity mediates aggression in choruses of the

678 Pacific treefrog. Animal Behaviour 47:633-41.

679 Brooks, R. J., and J. B. Falls. 1975a. Individual recognition by song in white-throated sparrows. I.

680 Discrimination of songs of neighbors and strangers. Canadian Journal of Zoology 53:879-888.

$681 ـ .1975 b$. Individual recognition by song in white-throated sparrows. III. Song features used in

682 individual recognition. Canadian Journal of Zoology 53:1749-61.

683 Burmeister, S. S., A. G. Ophir, M. J. Ryan, and W. Wilczynski. 2002. Information transfer during cricket

684 frog contests. Animal Behaviour 64:715-725.

685 Bush, S. L., H. C. Gerhardt, and J. Schul. 2002. Pattern recognition and call preferences in treefrogs

686 (Anura: Hylidae): a quantitative analysis using a no-choice paradigm. Animal Behaviour 63:7-14.

687 Carlson, N. V., E. M. Kelly, and I. Couzin. 2020. Individual vocal recognition across taxa: a review of the

688 literature and a look into the future. Philosophical Transactions of the Royal Society B: Biological

689 Sciences 375:20190479.

690 Caves, E. M., P. A. Green, M. N. Zipple, S. Peters, S. Johnsen, and S. Nowicki. 2018. Categorical

691 perception of colour signals in a songbird. Nature 560:365-367.

692 Cheney, D. L., and R. M. Seyfarth. 1980. Vocal recognition in free-ranging vervet monkeys. Animal

693 Behaviour 28:362-367.

694 Chuang, M.-F., Y.-C. Kam, and M. A. Bee. 2017. Territorial olive frogs display lower aggression towards

695 neighbours than strangers based on individual vocal signatures. Animal Behaviour 123:217-228.

696 Colgan, P. W. 1983. Comparative social recognition. Wiley.

697 Davies, N. B. 1978. Territorial defence in the speckled wood butterfly (Pararge aegeria): The resident 
always wins. Animal Behaviour 26:138-147.

699 Davis, M. S. 1987. Acoustically mediated neighbor recognition in the North American bullfrog, Rana

700 catesbeiana. Behavioral Ecology and Sociobiology 21:185-190.

701 Dyson, M. L., M. S. Reichert, and T. R. Halliday. 2013. Contests in amphibians. Pages 228-257 in I. C.

702 W. Hardy and M. Briffa, eds. Animal Contests. Cambridge University Press.

703 Edwards, C. J., T. B. Alder, and G. J. Rose. 2002. Auditory midbrain neurons that count. Nature

704 neuroscience 5:934-6.

705 Ehret, G. 1992. Categorical perception of mouse-pups ultrasounds in the temporal domain. Animal

706 Behavior 43:409-416.

707 Ehret, G., and B. Haack. 1981. Categorical perception of mouse pup ultrasound by lactating females.

708 Naturwissenschaften 68:208-209.

709 Engelbrecht-Wiggans, E., and J. P. Tumulty. 2019. "Reverse" sexual dichromatism in a Neotropical frog.

710 Ethology 125:957-964.

711 Gerhardt, H. C. 1991. Female mate choice in treefrogs: static and dynamic acoustic criteria. Animal

712 Behaviour 42:615-635.

713 1992. Multiple messages in acoustic signals. Seminars in the Neurosciences 4:391-400.

714 Gerhardt, H. C. 2001. Acoustic communication in two groups of closely related treefrogs. Advances in

715 the Study of Behavior 30:99-167.

716 Gerhardt, H. C., M. L. Dyson, and S. D. Tanner. 1996. Dynamic properties of the advertisement calls of 717 gray tree frogs: patterns of variability and female choice. Behavioral Ecology 7:7-18.

718 Gerhardt, H. C., and F. Huber. 2002. Acoustic Commmunication in Insects and Anurans. University of

719 Chicago Press, Chicago.

720 Gerhardt, H. C., J. D. Roberts, M. A. Bee, and J. J. Schwartz. 2000. Call matching in the quacking frog

721 (Crinia georgiana). Behavioral Ecology and Sociobiology 48:243-251.

722 Gerhardt, H. C., and J. Schul. 1999. A quantitative analysis of behavioral selectivity for pulse rise-time in 723 the gray treef rog, Hyla versicolor. J Comp Physiol A 185:33-40. 
724 Gescheider, G. A. 1976. Psychophysics. Methods and Theory. Lawrence Erlbaum Associates, Hillsdale, 725 NJ.

726 Getty, T. 1987. Dear enemies and the prisoner's dilemma: why should territorial neighbors form 727 defensive coalitions? American Zoologist 27:327-36.

728 . 1995. Search, discrimination, and selection: mate choice by pied flycatchers. The American

729 Naturalist 145:146-154.

730 Guilford, T., and M. S. Dawkins. 1991. Receiver psychology and the evolution of animal signals. Animal

731 Behaviour 42:1-14.

732 Hanley, D., A. V. López, V. D. Fiorini, J. C. Reboreda, T. Grim, and M. E. Hauber. 2019. Variation in

733 multicomponent recognition cues alters egg rejection decisions: A test of the optimal acceptance

734 threshold hypothesis. Philosophical Transactions of the Royal Society B: Biological Sciences 374.

735 Hauber, M. E., C. Moskát, and M. Bán. 2006. Experimental shift in hosts' acceptance threshold of

736 inaccurate-mimic brood parasite eggs. Biology Letters 2:177-180.

737 Humfeld, S. C., V. T. Marshall, and M. A. Bee. 2009. Context-dependent plasticity of aggressive

738 signalling in a dynamic social environment. Animal Behaviour 78:915-924.

739 Illes, A. E., M. L. Hall, and S. L. Vehrencamp. 2006. Vocal performance influences male receiver

740 response in the banded wren. Proceedings of the Royal Society B: Biological Sciences 273:1907-1912.

741 Kokko, H., A. López-Sepulcre, and L. J. Morrell. 2006. From hawks and doves to self-consistent games

742 of territorial behavior. American Naturalist 167:901-912.

743 Krebs, J. R. 1982. Territory defence in the great tit (Parus major): Do residents always win? Behavioral

744 Ecology and Sociobiology 11:185-194.

745 Ligon, R. A. 2014. Defeated chameleons darken dynamically during dyadic disputes to decrease danger

746 from dominants. Behavioral Ecology and Sociobiology 68:1007-1017.

747 Lynn, S. K., J. Cnaani, and D. R. Papaj. 2005. Peak shift discrimination as a mechanism of signal

748 evolution. Evolution 59:1300-1305.

749 Marshall, V. T., S. C. Humfeld, and M. A. Bee. 2003. Plasticity of aggressive signalling and its evolution 
in male spring peepers, Pseudacris crucifer. Animal Behaviour 65:1223-1234.

751 Miller, C. T., and M. A. Bee. 2012. Receiver psychology turns 20: is it time for a broader approach?

752 Animal Behaviour 83:331-343.

753 Mora-Kepfer, F. 2014. Context-dependent acceptance of non-nestmates in a primitively eusocial insect.

754 Behavioral Ecology and Sociobiology 68:363-371.

755 Myberg, A. A. J., and R. J. Riggio. 1985. Acoustically mediated individual recognition by a coral reef

756 fish (Pomacentrus partitus). Animal Behaviour 33:411-416.

757 Narins, P. M., S. W. F. Meenderink, J. P. Tumulty, A. Cobo-Cuan, and R. Márquez. 2018. Plant-borne

758 vibrations modulate calling behaviour in a tropical amphibian. Current Biology 28:R1333-R1334.

759 Nelson, D. A. 1988. Feature weighting in species song recognition by the field sparrow (Spizella pusilla).

760 Behaviour 106:158-181.

761 Nelson, D. A. 1989. Song frequency as a cue for recognition of species and individuals in the field

762 sparrow (Spizella pusilla). Journal of Comparative Psychology 103:171-6.

763 Nelson, D. A., and P. Marler. 1989. Categorical perception of a natural stimulus continuum: birdsong.

764 Science 244:976-978.

765 . 1990. The perception of birdsong and an ecological concept of signal space. Pages $443-478$ in

766 W. C. Stebbins and M. A. Berkley, eds. Comparative Perception: Vol II. Complex Signals. John Wiley \&

767 Sons, Inc, New York.

768 O’Connor, C. M., A. R. Reddon, I. Y. Ligocki, J. K. Hellmann, K. A. Garvy, S. E. Marsh-Rollo, I. M.

769 Hamilton, et al. 2015. Motivation but not body size influences territorial contest dynamics in a wild

770 cichlid fish. Animal Behaviour 107:19-29.

771 Owen, P. C., and S. A. Perrill. 1998. Habituation in the green frog, Rana clamitans. Behavioral Ecology

772 and Sociobiology 44:209-213.

773 Peeke, H. V. S. 1984. Habituation and the maintenance of territorial boundaries. Pages 393-421 in H. V.

774 S. Peeke and L. Petrinovich, eds. Habituation, Sensitization, and Behavior. Academic Press, Orlando.

775 Peeke, H. V., and S. C. Peeke. 1973. Habituation in fish with special reference to intraspecific aggressive 
behavior. Pages 59-83 inHabituation: Behavioral studies.

777 Petrinovich, L. 1984. A two-factor dual-process theory of habituation and sensitization. in H. V. S. Peeke

778 and L. Petrinovich, eds. Habituation, sensitization, and behaviour. Academic Press, New York.

779 Pettitt, B. A., G. R. Bourne, and M. A. Bee. 2012. Quantitative acoustic analysis of the vocal repertoire of

780 the golden rocket frog (Anomaloglossus beebei). The Journal of the Acoustical Society of America

$781 \quad 131: 4811-20$.

782 2013. Advertisement call variation in the golden rocket frog (Anomaloglossus beebei): evidence

783 for individual distinctiveness. Ethology 119:244-256.

784 Pettitt, B. A., G. R. Bourne, and M. A. Bee. 2018. Predictors and benefits of microhabitat selection for

785 offspring deposition in golden rocket frogs. Biotropica 50:919-928.

786 Pettitt, B. A., G. R. Bourne, and M. A. Bee. 2020. Females prefer the calls of better fathers in a

787 Neotropical frog with biparental care. Behavioral Ecology 31:152-163.

788 Platz, J. E., and D. C. Forester. 1988. Geographic variation in mating call among the four subspecies of

789 the chorus frog: Pseudacris triseriata (Wied). Copeia 1988:1062-1066.

790 Pollack, G. S., and R. R. Hoy. 1979. Temporal pattern as a cue for species-specific calling song

791 recognition in crickets. Science 204:429-432.

792 Proops, L., K. Mccomb, and D. Reby. 2009. Cross-modal individual recognition in domestic horses

793 (Equus caballus). Proceedings of the National Academy of Sciences 106:947-951.

794 R Core Team. 2017. R: A language and environment for statistical computing. R Foundation for

795 Statistical Computing, Vienna, Austria.

796 Rankin, C. H., T. Abrams, R. J. Barry, S. Bhatnagar, D. F. Clayton, J. Colombo, G. Coppola, et al. 2009.

797 Habituation revisited: An updated and revised description of the behavioral characteristics of habituation.

798 Neurobiology of Learning and Memory 92:135-138.

799 Reeve, H. K. 1989. The evolution of conspecific acceptance thresholds. The American Naturalist

$800 \quad 133: 407-435$.

801 Reichert, M. S. 2010. Aggressive thresholds in Dendropsophus ebraccatus: Habituation and sensitization 
to different call types. Behavioral Ecology and Sociobiology 64:529-539.

803 Rose, G. J. 2014. Time computations in anuran auditory systems. Frontiers in Physiology 5:1-8.

804 Rossi, N., D. Baracchi, M. Giurfa, and P. D’Ettorre. 2019. Pheromone-induced accuracy of nestmate

805 recognition in carpenter ants: simultaneous decrease in type I and type II errors. The American Naturalist

$806 \quad 193: 267-278$

807 Scharf, H. M., A. V. Suarez, H. K. Reeve, and M. E. Hauber. 2020. The evolution of conspecific

808 acceptance threshold models. Philosophical Transactions of the Royal Society B: Biological Sciences

$809 \quad 375: 20190475$.

810 Shettleworth, S. J. 2010. Cognition, evolution, and behavior. Oxford University Press, New York, NY.

811 Siracusa, E., S. Boutin, M. M. Humphries, J. C. Gorrell, D. W. Coltman, B. Dantzer, J. E. Lane, et al.

812 2017. Familiarity with neighbours affects intrusion risk in territorial red squirrels. Animal Behaviour

$813 \quad 133: 11-20$.

814 Steiger, S., and J. K. Müller. 2010. From class-specific to individual discrimination: Acceptance threshold

815 changes with risk in the partner recognition system of the burying beetle Nicrophorus vespilloides.

816 Animal Behaviour 80:607-613.

817 Stoddard, P. K. 1996. Vocal recognition of neighbors by territorial passerines. Pages 356-34 in D. E.

818 Kroodsma and E. H. Miller, eds. Ecology and Evolution of Acoustic Communication in Birds. Cornell

819 University Press, Ithaca, NY.

820 Stratton, G. E., and G. W. Uetz. 1983. Communication via substratum-coupled stridulation and

821 reproductive isolation in wolf spiders (Araneae: Lycosidae). Animal Behaviour 31:164-172.

822 Sumner, C. J., and S. Sumner. 2020. Signal detection: applying analysis methods from psychology to

823 animal behaviour. Philosophical Transactions of the Royal Society B: Biological Sciences 375:20190480.

824 Tanner, J. C., and J. P. Tumulty. 2020. The signals and category boundaries that enable categorical

825 perception: a comment on Green et al. Behavioral Ecology 31:871-871.

826 Tanner, J. C., J. L. Ward, R. G. Shaw, and M. A. Bee. 2017. Multivariate phenotypic selection on a 827 complex sexual signal. Evolution 71:1742-1754. 
828 Temeles, E. 1994. The role of neighbours in territorial systems: when are they "dear enemies"? Animal

829 Behaviour 47:339-350.

830 Tibbetts, E. A., and J. Dale. 2007. Individual recognition: it is good to be different. Trends in Ecology \&

831 Evolution 22:529-37.

832 Tumulty, J. P. 2018. Dear Enemy Effect. Pages 1-4 in J. Vonk and T. Shackelford, eds. Encyclopedia of

833 Animal Cognition and Behavior. Springer International Publishing, Cham.

834 Tumulty, J. P., and M. A. Bee. 2021. Ecological and social drivers of neighbor recognition and the dear

835 enemy effect in a poison frog. Behavioral Ecology 32:138-150.

836 Tumulty, J. P., Z. K. Lange, and M. A. Bee. 2021. Identity signaling, identity reception, and the evolution

837 of social recognition in a Neotropical frog. bioRxiv.

838 Tumulty, J. P., and M. J. Sheehan. 2020. What drives diversity in social recognition mechanisms?

839 Frontiers in Ecology and Evolution 7:517.

840 Vélez, A., W. Hödl, and A. Amézquita. 2012. Sound or silence: call recognition in the temporal domain

841 by the frog Allobates femoralis. Ethology 118:377-86.

842 Wells, K. D., and J. J. Schwartz. 1984. Vocal communication in a neotropical treefrog, Hyla ebraccata:

843 aggressive calls. Behaviour 91:128-145.

844 Wiley, R. H. 1994. Errors, exaggeration, and deception in animal communication. Pages 157-189 in L. A.

845 Real, ed. Behavioral Mechanisms in Evolutionary Ecology. University of Chicago Press, Chicago \&

846 London.

847 2006. Signal detection and animal communication. Advances in the Study of Behavior 36:217-

848247.

$849-2013 a$. Specificity and multiplicity in the recognition of individuals: implications for the

850 evolution of social behaviour. Biological Reviews 88:179-95.

$851-2013 \mathrm{~b}$. A receiver-signaler equilibrium in the evolution of communication in noise. Behaviour

$852 \quad 150: 957-993$.

853 Wilson, E. O. 1975. Sociobiology: The New Synthesis. Harvard University Press, Cambridge, MA. 
854 Wrangham, R. W. 1999. Evolution of coalitionary killing. Yearbook of Physical Anthropology 42:1-30.

855 Yang, Y., V. Prémel, and C. L. Richards-Zawacki. 2020. Prior residence effect determines success of

856 male-male territorial competition in a color polymorphic poison frog. Ethology 126:1131-1140.

857 Yorzinski, J. L. 2017. The cognitive basis of individual recognition. Current Opinion in Behavioral

858 Sciences 16:53-57.

859 